\title{
Telegenetics use in presymptomatic genetic counselling: patient evaluations on satisfaction and quality of care
}

\author{
Ellen Otten ${ }^{\star, 1}$, Erwin Birnie ${ }^{1}$, Adelita V Ranchor ${ }^{2}$ and Irene M van Langen ${ }^{\star, 1}$
}

In recent years, online counselling has been introduced in clinical genetics to increase patients' access to care and to reduce time and cost for both patients and professionals. Most telegenetics reports so far evaluated online oncogenetic counselling at remote health centres in regions with large travelling distances, generally showing positive patient outcomes. We think online counselling - including the use of supportive tools that are also available during in-person counselling - of presymptomatic patients in their homes can also be feasible and valuable for patients in relatively small regions. We performed a single-centre pilot study of online genetic counselling for 57 patients who were presymptomatic cardiogenetic $(n=17)$, presymptomatic oncogenetic $(n=34)$ and prenatal ( 3 couples). One-third of presymptomatic patients we approached consented to online counselling. Patient evaluations of practical aspects, satisfaction and psychological outcomes were assessed and compared with a matched control group. Patients managed to fulfil the preparations, were significantly more satisfied with their counsellor and counselling session than controls and were satisfied with the online counselling more than they expected to be beforehand. Psychological outcomes (decreased anxiety and increased control) did not differ with control patients. Technical problems occurred in almost half of online sessions. Nonetheless, online counselling in patients' homes proved to be feasible and was appreciated by a substantial part of presymptomatic patients at our genetics centre in the Netherlands. Based on these outcomes, we conclude online counselling can be a valuable addition to existing counselling options in regular patient care. European Journal of Human Genetics (2016) 24, 513-520; doi:10.1038/ejhg.2015.164; published online 15 July 2015

\section{INTRODUCTION}

Telemedicine applications have recently been introduced in clinical genetics (generally referred to as telegenetics), meaning the remote counselling or evaluation of patients in real time by video and audio applications. The underlying reasons for its introduction concern increased access and efficiency of care.

Currently, genetics departments are facing efficiency measures, cost reductions and shortage of clinical genetic professionals. Simultaneously, the widespread introduction of next-generation sequencing is increasing the number of requests for and yield of genetic counselling and testing. Literature reports suggest that telegenetic care can be useful, patient-friendly, effective and psychologically acceptable for various indications in the light of economic and personnel scarcity on the one hand, and increasing awareness and counselling requests from patients spread over a large geographical area on the other hand. ${ }^{1-7}$

So far, almost all reports of telegenetics programmes described counselling between professionals at a central clinic and patients at regional clinics, with a genetic counsellor present, in regions with large travelling distances. ${ }^{1-6,8-11}$ Our pilot project differs from previous reports by (1) counselling sessions taking place in patients' homes, (2) covering patients in a non-remote area and (3) having cardiogenetic as well as previously reported oncogenetic and prenatal counselling. We think the appliance of telegenetics could also be successful in relatively small countries in the aforementioned respects and, moreover, that online counselling from patients' homes without a counsellor on site could be feasible and advantageous for some of our patients. Therefore, we evaluated our application for online genetic counselling from the patients' perspective. It included various supportive tools that are also available during in-person genetic counselling, for example, simultaneously viewing disease information brochures or supportive drawings and the webcam image of the involved patient and counsellor on-screen. We evaluated the quality of care and patients' satisfaction with the counselling sessions and counsellors: Changes in psychological outcomes and satisfaction with counselling of online patients should be at least similar, on average, to those of controls given regular counselling at our outpatient department (OPD). The satisfaction with online counselling should, on average, be at least equal to patients' expectations, with a moderate or high satisfaction level. This would be in line with the generally reported mean satisfaction level of patients with counselling $(\geq 7$ on a $1-10$ scale). As part of our evaluation, we also assessed patients' opinions about saliva self-collection for DNA testing.

\section{MATERIALS AND METHODS}

Online counselling application

The online counselling application used in our study was established in association with the 'myCoachconnect' company (www.mycoachconnect.com). This secure platform contains several functions in addition to videoconferencing, which aim to support the information exchange during counselling and to

\footnotetext{
${ }^{1}$ University of Groningen, University Medical Centre Groningen, Department of Genetics, Groningen, The Netherlands; ${ }^{2}$ Department of Health Psychology, University Medical Centre Groningen, University of Groningen, Groningen, The Netherlands

${ }^{*}$ Correspondence: Dr E Otten or Professor IM van Langen, University of Groningen, University Medical Center Groningen, Department of Genetics, PO Box 30001, Groningen 9700 RB, The Netherlands. Tel: +31 50361 7229; Fax: +31 50361 7231; E-mail: e.otten@umcg.nl or i.m.van.langen@umcg.nl

Received 13 February 2015; revised 7 May 2015; accepted 27 May 2015; published online 15 July 2015
} 
allow future access for patients to this information. These include a notepad for counsellors and notebook for patients, transfer and viewing of disease information brochures and visiting websites together during counselling, and a secured email and patient registration/invitation system (Supplementary Material and Supplementary Figures 1a-f). The application satisfied the safety protocols of our university medical centre (UMC). Privacy protocols were drawn up in consultation with the centre's ICT and personal privacy protection advisors.

\section{Setting and study design}

We offered online genetic counselling in the region of northern Netherlands covered by our department at the UMC Groningen. It has about 6000 new referrals each year, with a mean annual increase of $6 \%$ in recent years. Regular outpatient clinics are held in four regional hospitals at a mean distance of $78 \mathrm{~km}$. We performed a single-centre matched cohort study, with pre- and postcounselling measurements. We matched by sex and indication (frequency matching), and compared the expectations and outcomes of 57 patients receiving online counselling with those of 71 controls receiving in-person counselling for cardiogenetic and oncogenetic cascade screening for a known familial mutation, and urgent prenatal counselling and testing. Other inclusion criteria were as follows: (1) an appointment comprising a full genetic counselling session; (2) planned at least two days after referral; (3) a maximum of two participants per session on the patient's side; (4) access to a computer with a webcam and internet (for online patients); and (5) verbal consent given by online patients during the first telephone contact and digital consent by creating a personal account in our online platform. Delivery of DNA testing results for both the online and control group was in accordance with the applicable standard for result delivery for each separate indication and test outcome (carrier or non-carrier), and the possible preferences of the involved counselor and/or patient. The study protocol was reviewed by our institutional ethics committee, who found formal ethical consideration not required.

\section{Online patients and procedures}

We included online patients between August 2011 and April 2012. The online group was completed first and then the control patients were included until matching to the online group was achieved. Patients were informed by telephone about the study and were asked whether they would be interested in online counselling. Pre-counselling contact with those who agreed included the following (Figure 1a): (1) an email with appointment information, a log-in for the online platform, a telephone number and email address for questions and practical support, and a hyperlink to an instruction video about the online counselling process; (2) an email with a short digital form for family information to complete and return, after patients confirmed their account; (3) telephone contact with a case manager a few days before the session to check their connection and access to the online counselling. Patients were also sent online pre- and postcounselling study questionnaires, and a paper laboratory form with a saliva self-collection kit, which was only suitable for testing of mutations or small deletions. If patients had to be tested for a larger deletion, they were sent a laboratory form for blood withdrawal locally after counselling.

\section{Control patients}

Control patients who had conventional counselling at one of our OPDs and had not previously been invited to participate in the online counselling group were included between December 2011 and December 2012 (oncogenetic cardiogenetic), and March to April 2013 (prenatal). In the overlapping period (December 2011 to April 2012), we alternated recruiting online and control patients weekly. Controls were sent online study questionnaires, but for logistical reasons prenatal controls were asked to complete paper questionnaires immediately before counselling and within a couple of days afterwards.

\section{Patient measurements}

The online study questionnaires for both groups had to be completed 1 week before and within a couple of days after counselling, and covered the following measurements (Table 1).
Patient characteristics. Data on patients' age (in years), sex, educational level (low/high) and the indication for genetic counselling (cardiogenetic/oncogenetic/prenatal) were recorded. The patients' experience with computers, internet and online communication were assessed by various statements on a five-point Likert scale (range: 'totally disagree' to 'totally agree') and by their answers to multiple-choice questions on their frequency of use and experience with online communication.

Satisfaction with counselling and content. Patients' satisfaction with genetic counselling was measured using the seven-item Clinical Genetics Satisfaction (CGS) indicator, with a 1-5 Likert scale response mode. Higher scores indicate greater satisfaction. The Dutch Clinical Genetics Association adopted the CGS. The English version shows excellent internal consistency in a clinical genetic setting $(\alpha=0.91) .{ }^{12}$ The internal consistency in our present study was 0.98 . Patients also had to indicate how far 10 aspects of the genetic disorder concerned were addressed during their counselling session (5-point Likert scale; range 'far too little' to 'far too much').

Psychological measures. We used the validated Dutch nine-item version of the Perceived Personal Control (PPC) questionnaire to assess perceived control of our patients before and after counselling $(\alpha=0.79-0.81)^{13}$ on a $0-2$ Likert scale. Higher scores indicate greater levels of perceived control. We used the Dutch six-item version of the State and Trait Anxiety Inventory (STAI) to assess experienced anxiety by patients before and after counselling, on a 1-4 Likert scale $(\alpha=0.83) .{ }^{14}$ The STAI has been validated in a clinical genetic setting $(\alpha=0.82) .{ }^{15}$ Higher scores indicate greater levels of anxiety.

Expectations and evaluation of online counselling. All patients' expectations of online counselling were rated by various statements (eg, wanting to see the counsellor in-person after online counselling, intending to use online counselling more often in the future) on a five-point Likert scale (range 'totally disagree' to 'totally agree'). They were also asked to describe their expected advantages/disadvantages of online versus in-person counselling (eg, time saving, feeling more/less at ease and being more/less focussed on the counselling). Afterwards, online patients were asked about these aspects again and also to indicate whether and how online counselling had to be improved to make it equivalent to OPD counselling, to weigh the advantages of online counselling against its disadvantages and to give an overall judgement of online counselling on a scale of $1-10$ (10 being most positive).

Telemedicine satisfaction. The Telemedicine Satisfaction Questionnaire (TSQ) was used to measure expected satisfaction with Telemedicine for all patients and online patients' perceived satisfaction. ${ }^{16}$ Higher scores (Likert scale 1-5) indicate greater satisfaction, with 4 representing moderately satisfied and 5 highly satisfied. We translated the validated 14-item English TSQ into Dutch in a validated way, using 'back translation'. We removed item 7 ('I think the healthcare provided via telemedicine is consistent'), because it did not apply to our setting. We adapted the item formulation so that we could use the TSQ both before and after counselling. We calculated and compared mean item scores before and afterwards, and had internal consistencies of 0.88 and 0.72 , respectively.

Evaluation of practical issues and responsibilities. Patients were asked several multiple-choice questions about their experiences with preparing for online counselling (eg, clearness of the instruction emails and movie, and preparation time required), the occurrence of technical problems during the session (eg, problems occurred yes/no and temporary/continuous, and distracting from counselling yes/no) and their acceptance of patient responsibility for various aspects of online counselling: planning an appointment, preparation before counselling, proper functioning of the application during the session and initiating DNA testing (1-5 Likert scale; range 'not acceptable at all' to 'very acceptable').

\section{Data analysis}

For normally distributed variables we used mean (SD). Median (IQR) was used for variables with skewed distribution and $n(\%)$ was used for both ordinal and nominal variables. Mean item scores per patient were calculated for the questionnaires PPC, STAI, CGS and TSQ, if at least two-thirds of each were completed. Adjusted mean differences between groups were estimated with linear regression analysis, with mean item score as dependent variable, and 
a online counselling process

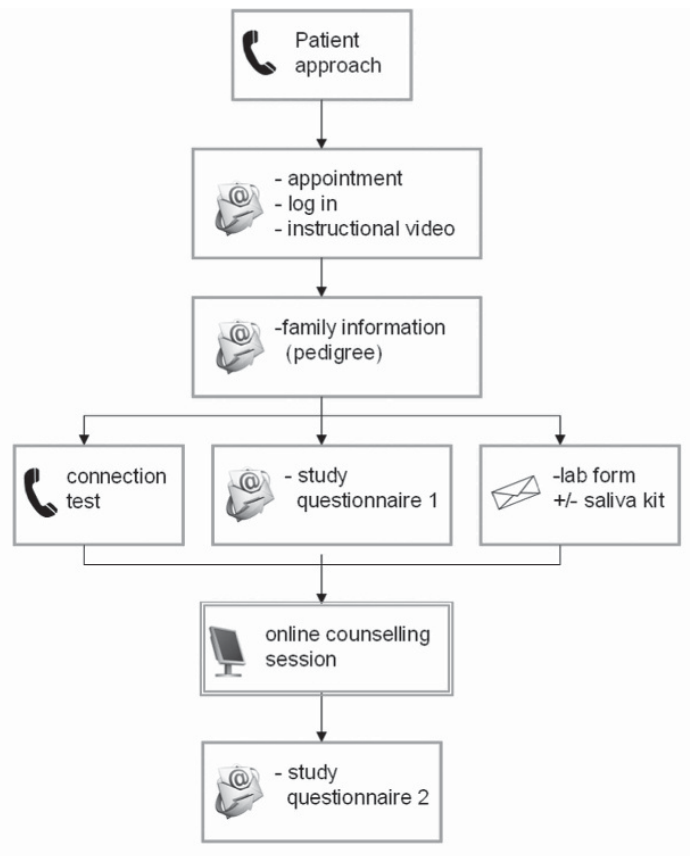

b patient uptake when offered online counselling

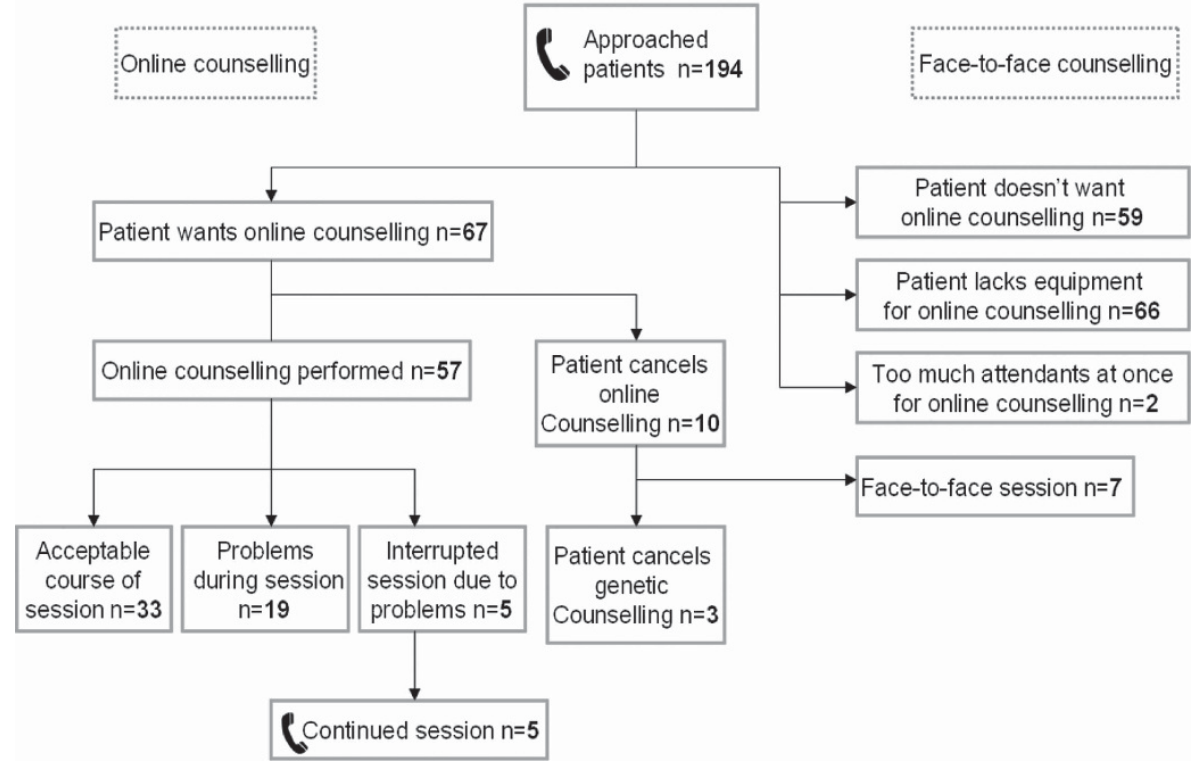

Figure 1 Flow schemes for the online counselling process. (a) Online counselling process. (b) Patient uptake when offered online counselling.

gender and indication (matching factors), group (online/control) and educational level as independent variables. We calculated effect sizes by dividing the difference of mean scores over time or between groups, by the mean SD of both time points or the pooled SD of both groups. We used paired samples and independent sample $t$-tests to analyse outcomes of normally distributed variables and $\chi^{2}$-test or Fisher's exact test for outcomes of all categorical variables.

\section{RESULTS}

Patient outcomes

Of the patients we approached, $35 \%(n=67)$ chose for online counselling (Figure 1b); 65\% were excluded, because they lacked the proper equipment for online counselling $(34 \%, n=66$, mean age 51.5 years, $44 \%$ male) or preferred in-person counselling $(31 \%, n=59$, mean age 47.2 years, $44 \%$ male). Eventually, 57 patients (mean age 44 years, $53 \%$ male) had online counselling. Ten patients (mean age 42.9 years, 20\% male) cancelled their appointment for various reasons: no longer interest in counselling and testing $(n=3)$, prefer OPD session on second thought $(n=2)$, join a relative or quicker appointment elsewhere $(n=3)$, or technical problems with home equipment $(n=2)$. Of 102 OPD patients invited to complete the online preand postcounselling survey, 69.6\% $(n=71)$ cooperated and was included in the control group.

Patient characteristics. The online counselling group comprised 57 patients (51 counselling sessions) of which 17 had cardiogenetic 
(cardiomyopathy and long QT syndrome) and 34 had oncogenetic counselling (BRCA1/2, Lynch syndrome). Three couples (six patients) had genetic counselling during pregnancy because of fetal anomalies. Online patients and controls did not differ significantly regarding sex, age, educational level or indication for counselling (Table 2). There were also no significant differences between the groups for reported frequency of computer use, experience with online communication for work and/or private purposes, frequency of online communication for work and/or private purposes, and experience with online communication with healthcare professionals other than by email. However, before their counselling session, significantly more patients in the online group had communicated by email with healthcare professionals in general compared with controls (25 versus $10 \%$; $P=0.047$ ) and they seemed to be more open and comfortable overall in using and extending their computer use. The mean scores for all related questionnaire items differed significantly between the two groups (Supplementary Table S1).

Satisfaction with counselling (CGS indicator) and content. Online patients had a significantly higher mean CGS item score (reflecting greater satisfaction) after counselling than controls (Table 3a). However, satisfaction with the counselling content did not differ significantly between the two groups: mean item scores were 2.96 (SD 0.12) for online patients and 2.91 (SD 0.30) for controls. This indicates that both groups found that all aspects of the heritable disease concerned were sufficiently discussed overall.

Psychological outcomes. The mean change of PPC item score for online patients (after the counselling session compared with beforehand) did not differ significantly from controls (Table $3 b$ ). The mean PPC item

Table 1 Overview of questionnaire designs

\begin{tabular}{|c|c|c|c|c|}
\hline Measurement instrument/aspect & $\begin{array}{l}\text { Online } \\
\text { before }\end{array}$ & $\begin{array}{l}\text { Online } \\
\text { after }\end{array}$ & $\begin{array}{l}\text { Control } \\
\text { before }\end{array}$ & $\begin{array}{c}\text { Control } \\
\text { after }\end{array}$ \\
\hline Personal information & $x$ & $x$ & $x$ & $x$ \\
\hline $\begin{array}{l}\text { PC/internet/online consulting } \\
\text { experience }\end{array}$ & $x$ & $x$ & $x$ & \\
\hline Online counselling, general & $x$ & $x$ & & $x$ \\
\hline $\begin{array}{l}\text { Practical aspects of online } \\
\text { counselling }\end{array}$ & & $x$ & & \\
\hline Patient responsibilities & & $x$ & & $x$ \\
\hline TSQ & $x$ & $x$ & & $x$ \\
\hline PPCa & $x$ & $x$ & $x$ & $x$ \\
\hline STAl $^{a}$ & $x$ & $x$ & $x$ & $x$ \\
\hline CGS $^{a}$ & & $x$ & & $x$ \\
\hline Content of counselling session & & $x$ & & $x$ \\
\hline
\end{tabular}

Abbreviations: CGS, Clinical Genetics Satisfaction indicator; PPC, Personal Perceived Control questionnaire; STAI, State-Trait Anxiety Inventory; TSQ, Telemedicine Satisfaction Questionnaire.

aStandard validated questionnaire.

Table 2 Characteristics of patient groups

\begin{tabular}{lcc}
\hline & Online & Control \\
\hline Number of patients & 57 & 71 \\
Sex; \% male/female & $53 / 47$ & $51 / 49$ \\
Mean age in years (range) & $44(22-74)$ & $47(19-74)$ \\
Educational level; \% low/high & $51 / 49(4 \mathrm{Missings})$ & $68 / 32(2 \mathrm{Missings})$ \\
Indication: \% cardiac/oncology/prenatal & $30 / 60 / 10$ & $24 / 62 / 14$ \\
\hline
\end{tabular}

score for both online and control patients was significantly higher after counselling than beforehand. The respective effect sizes were of moderate magnitude. The mean change of STAI item score after counselling compared with beforehand did not differ significantly between online and control patients. Although the STAI mean item scores only changed significantly for the control group and not for the online group, the effect sizes were both $<0.20$, indicating a negligible change in both groups. Notably, the mean STAI item scores of online patients before and after counselling were significantly lower than those of controls. Scores of online patients having and not having technical problems during their session did not differ significantly with respect to their mean CGS item score and mean change of PPC and STAI scores.

\section{Expectations and evaluation of online counselling}

Expectations of online counselling. Online patients' scores on their expectations and experiences on various aspects of online counselling were significantly higher after compared with before their session on feeling comfortable with online counselling and the content delivery during the session, and significantly lower on wishing to see their counsellor in an additional OPD session (Table 4).

Expected advantages of online counselling mostly mentioned by 42 online patients were as follows: less time/travelling needed $(n=40)$, lower cost $(n=6)$, increased flexibility $(n=5)$, being in familiar environment/being more relaxed $(n=4)$ and the possibility of recording the session and the online availability of information $(n=4)$. Fifteen online patients expected disadvantages of online counselling beforehand, mostly being less personal contact due to the literal distance between the counsellor and patient $(n=11)$, less non-verbal communication $(n=3)$ and dependence on technology functioning well $(n=4)$.

Evaluations of online counselling. After their session, the most raised advantage again was less time/travelling needed $(n=43)$. The advantages of familiar environment $(n=12)$, saving cost $(n=9)$, possibility of recording/online availability of information $(n=4)$ and no need to take a day off or to arrange a babysitter $(n=3)$ were all mentioned more often after counselling than beforehand. Flexibility $(n=3)$ was mentioned less often. Afterwards, 28 patients indicated disadvantages such as lack of personal contact $(n=18)$, dependence on technology $(n=12)$, installation/complexity of the application $(n=2)$ and less non-verbal communication $(n=2)$.

After the counselling session, online patients rated the relative weight they assigned to the advantages and disadvantages of online counselling ( 1 = highly disadvantageous and $10=$ highly advantageous), and their mean overall score for the session on a $1-10$ scale. Mean scores were 8.4 (SD 1.05, range 5-10) and 8.3 (SD 0.95, range 6-10), respectively; perceived advantages overweighed the disadvantages of online counselling and patients were satisfied with their session overall. All patients reported having had enough opportunity to ask questions during the counselling, and $85 \%$ would recommend online counselling to friends (mean scores 4.94 (SD 0.24, $n=52$ ) and 4.54 (SD 0.75, $n=52$ ), respectively, scale 1-5). All online patients' suggestions for improvement were on technical aspects, for example, more stable internet connections, less sound delay and better image quality.

Telemedicine satisfaction. The mean TSQ item score of online patients before counselling (indicating their expected satisfaction with telemedicine) was significantly higher than that of controls. Online patients were also significantly more satisfied after their session than they had expected to be beforehand (Table $3 a$ and b, and Figures $2 \mathrm{a}$ and $\mathrm{b}$ ). Of online patients, $72 \%$ had an increased mean 
Table 3 Patient satisfaction and psychological outcomes: differences within and between both patient groups

a. Differences between groups

\begin{tabular}{|c|c|c|c|c|c|c|}
\hline TSQ before & $4.11(0.60 ; 47)$ & $2.84(0.90 ; 58)$ & $1.21(0.16)$ & $1.28(0.15)$ & $<0.001$ & 1.66 \\
\hline TSQ after & $4.52(0.40 ; 47)$ & $x$ & $\mathrm{x}$ & $\mathrm{x}$ & $\mathrm{x}$ & $x$ \\
\hline PPC before & $1.15(0.51 ; 46)$ & $1.07(0.46 ; 58)$ & $0.11(0.10)$ & $0.08(0.10)$ & 0.44 & 0.17 \\
\hline PPC after & $1.45(0.50 ; 46)$ & $1.29(0.46 ; 58)$ & $0.19(0.10)$ & $0.17(0.10)$ & 0.078 & 0.33 \\
\hline PPC change & $0.30(0.52 ; 46)$ & $0.21(0.36 ; 58)$ & 0.07 (0.09) & $0.09(0.09)$ & 0.28 & 0.21 \\
\hline STAI after & $1.64(0.55 ; 46)$ & $1.88(0.59 ; 58)$ & $-0.171(0.114)$ & $-0.24(0.11)$ & 0.033 & 0.42 \\
\hline STAI change & $0.10(0.43 ; 46)$ & $0.10(0.35 ; 58)$ & $-0.03(0.08)$ & $-0.003(0.08)$ & 0.97 & 0.008 \\
\hline CGS after & $4.91(0.23 ; 53)$ & $4.66(0.91 ; 61)$ & $0.27(0.14)$ & $0.25(0.12)$ & 0.040 & 0.42 \\
\hline
\end{tabular}

b. Differences within groups over time

\begin{tabular}{|c|c|c|c|c|}
\hline & Mean change (n) & $S D$ & Significance & Effect size \\
\hline TSQ change online & $0.41(47)$ & 0.57 & $<0.001$ & 0.82 \\
\hline PPC change online & $0.30(46)$ & 0.52 & $<0.001$ & 0.59 \\
\hline PPC change controls & $0.21(58)$ & 0.36 & $<0.001$ & 0.46 \\
\hline STAI change online & $0.10(46)$ & 0.43 & 0.132 & 0.18 \\
\hline STAI change controls & $0.10(58)$ & 0.35 & 0.033 & 0.17 \\
\hline
\end{tabular}

Abbreviations: CGS, Clinical Genetics Satisfaction indicator; PPC, Personal Perceived Control questionnaire; STAI, State-Trait Anxiety Inventory; TSQ, Telemedicine Satisfaction Questionnaire. a) All outcomes were tested by independent sample $t$-test; significant differences between groups are shown in bold, significant change $P<0.05$. Effect size is difference of mean scores between

groups divided by the pooled SD.
b) All outcomes were tested by paired samples $t$-test; significant changes pre-/post-counselling within groups are shown in bold, significant change $P<0.05$. Effect size is the difference of mean scores over time divided by the mean SD of both time points.

${ }^{a}$ Adjusted mean differences were measured by linear regression, adjusted for sex, counselling indication, educational level and counselling type (online versus in-person at outpatient department).

Table 4 General expectations and experiences of online counselling

\begin{tabular}{|c|c|c|c|c|}
\hline Comfortable with online talking about genetic testing $(n=48)$ & $3.94(0.84)$ & $4.63(0.76)$ & $0.68(8.83)$ & $<0.001$ \\
\hline Wish to see counsellor in-person on second occasion $(n=48)$ & $3.02(0.91)$ & $2.17(1.14)$ & $0.85(1.22)$ & $<0.001$ \\
\hline Good emotional interaction during counselling session $(n=48)$ & $3.98(0.86)$ & $4.25(0.98)$ & $0.27(1.25)$ & 0.14 \\
\hline Equality of online counselling with in-person counselling $(n=48)$ & $3.69(0.93)$ & $4.04(1.07)$ & $0.35(1.28)$ & 0.061 \\
\hline
\end{tabular}

amean score: $1=$ totally disagree, to $5=$ totally agree; changes measured by $t$-test.

${ }^{\text {b }}$ Significant changes are shown in bold, significant change $P<0.05$.

score after counselling compared with beforehand. In addition, 91\% had a mean itemscore $\geq 4$ afterwards $(n=47)$, indicating moderate to high satisfaction. Mean TSQ item score after counselling did not differ between those who had experienced technical problems during their session and those who did not.

The lowest scoring TSQ items, both beforehand and afterwards, were item 4 (seeing my healthcare provider as if in-person; mean score 3.49 and 3.87, respectively) and item 7 (obtaining better access to healthcare by telemedicine; mean score 3.68 and 3.49, respectively). The highest scoring items at both time points were item 8 (telemedicine saves time travelling; mean score 4.70 and 4.87, respectively) and item 9 (receiving adequate attention; mean score 4.45 and 4.91 , respectively).

Evaluation of technical issues, DNA-testing and patient responsibilities. Thirty-seven per cent of patients ( 21 of 57) reported technical problems during online counselling: These could be fixed completely during the session for five patients and partly for four patients. For eight patients the problems could not be fixed, but the session could nevertheless be continued, whereas for four patients the session had to be broken off because of the problems. Some (7 of 21) indicated that the problems distracted them from the counselling. Most patients $(n=9,47 \%)$ could fix the problems together with their counsellor. Help from a family member $(n=1,5 \%)$, from an employee of the genetics department $(n=3,16 \%)$ and fixing the problems by themselves $(n=2$, $11 \%)$ were also reported. Most patients who had technical problems during their online session indicated being satisfied with the degree of support in preparing for the session (instruction emails (80\%) and movie ( $88 \%)$ were clear) and managed well in fulfilling the preparations ( $85 \%$ did it all by themselves and $90 \%$ needed less than $10 \mathrm{~min}$ ).

Regarding their experience with saliva collection for DNA testing, most (10/11) online patients reported it was easy to do and the kit contained clear instructions. Seven patients would choose DNA testing in saliva over blood in the future, because of the ease of doing it at home in their own time. Two patients would prefer giving a blood sample the next time for fear of doing something wrong and thinking that blood testing was a more exact technique than DNA testing in saliva. 
a Before counselling $(\mathrm{n}=47)$

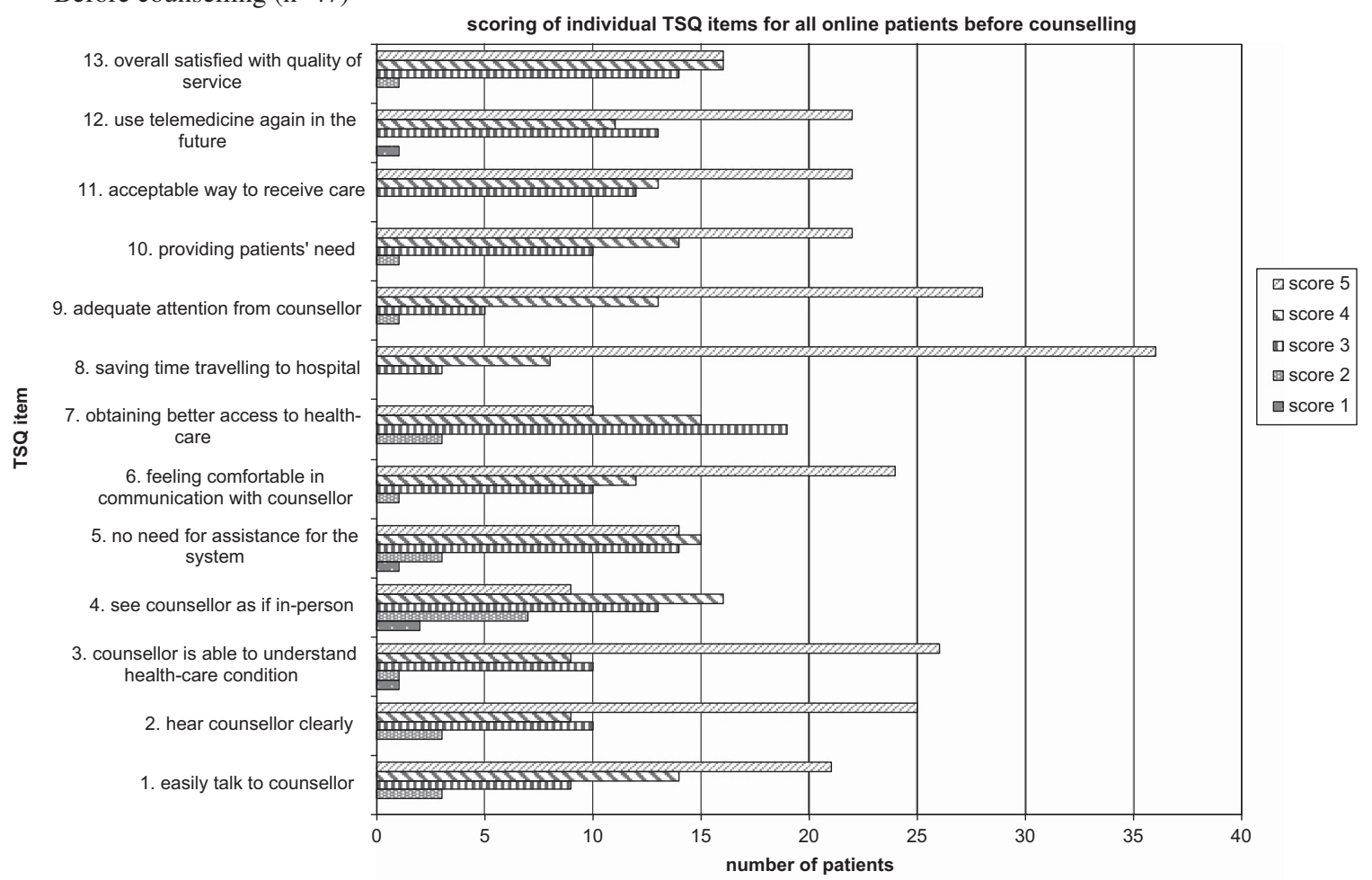

b After counselling $(n=47)$
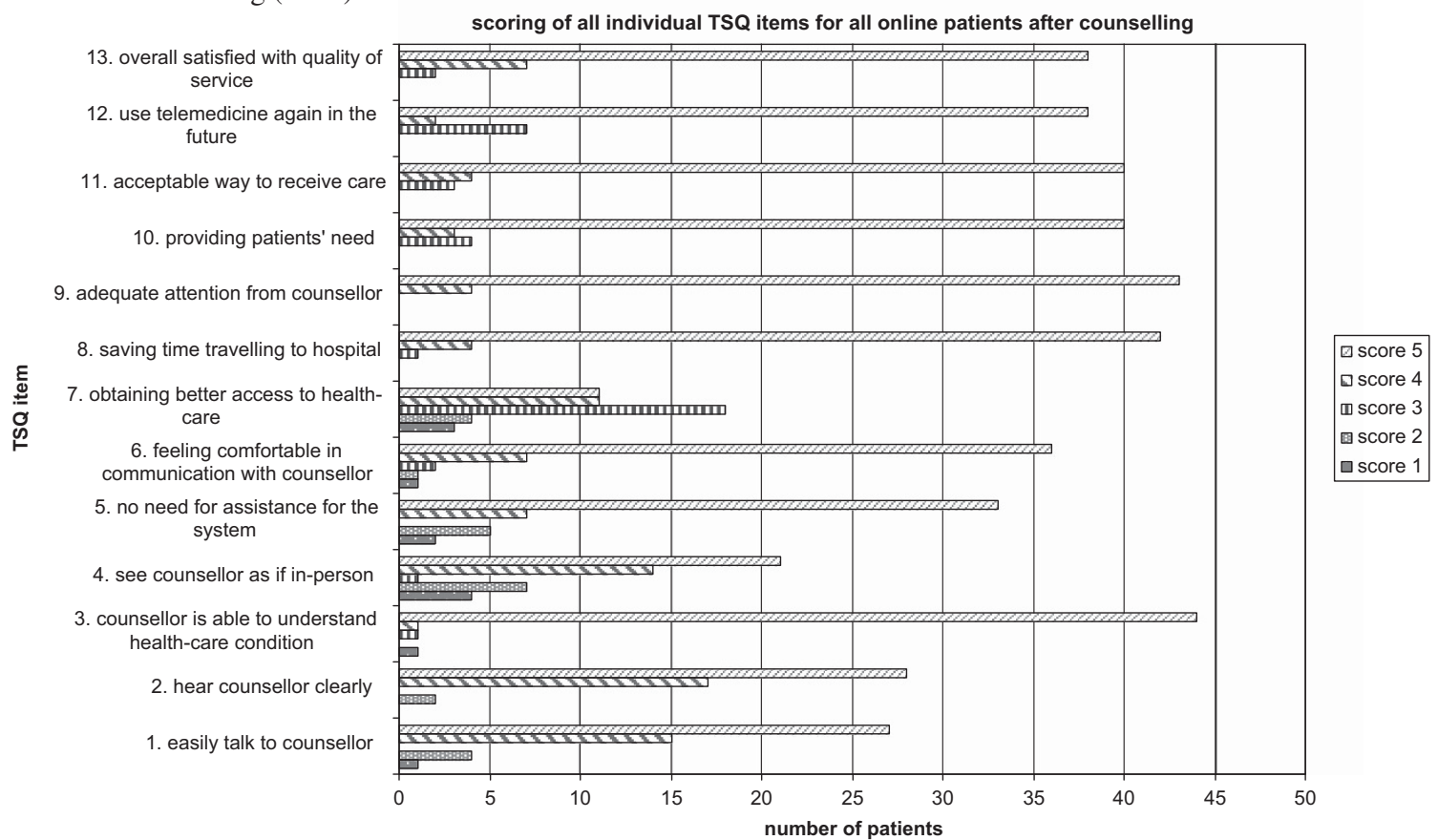

Figure 2 Scores by online patients for individual Telemedicine Satisfaction Questionnaire (TSQ) items. (a) Before counselling $(n=47)$. (b) After counselling $(n=47)$.

All items addressing the acceptability of patient responsibility in preparing and performing an online counselling session were judged significantly more positively by online patients (after their session) compared with controls (range $82-96 \%$ versus $53-57 \%$ acceptability; $P<0.001)$.

\section{DISCUSSION}

Overall, the criteria we set for patient outcomes in our study were met or even exceeded: patients who chose online counselling were satisfied with it, using our application with supportive tools. Moreover, their psychological outcomes (increased control and decreased anxiety) 
were comparable to controls and their satisfaction level with telemedicine was high, even higher than their positive expectations were beforehand. Despite having similar levels of computer experience, they seemed significantly more open and comfortable in using their computer and in extending its use than controls. They also had lower anxiety levels before and after counselling, and had significantly higher expectations of telegenetics than controls. Technical problems were reported by several online patients, but could mostly be fixed at the beginning or during the session, or the counselling session could still be completed despite the problems.

About one-third of approached presymptomatic patients consented to an online counselling session and were overall satisfied with the online counselling afterwards. One-third of approached patients could not be included, because they lacked the necessary equipment for online counselling. This uptake is comparable with a recent survey of Gardner et $a l^{17}$ on patient opinions regarding videoconsultations in patients' homes. In addition, Van de Belt et a ${ }^{18}$ recently reported that $21 \%$ of the general Dutch population would like to communicate with a health care provider via a webcam. These numbers will probably increase in the near future, as people become more used to online services and new devices are generally equipped with a webcam. Our report and review of the literature indicate that patients who have used online consulting applications are satisfied with it.

Our patients' preference for online counselling was not limited to a certain age group or gender, and online patients' satisfaction with telemedicine was significantly higher than their expectations before counselling. This trend or expectation has also been suggested in the literature for professionals, ${ }^{19,20}$ and similar outcomes can be expected for future patients who are initially reluctant to use telegenetics. For patients who are unable to use telegenetics, one could think of alternative options: performing online counselling at a location near the patient's home (eg, at their general practitioner's practice) or temporarily supplying patients with webcams on making an appointment for online counselling. Several authors have warned to be cautious with the overall positive outcomes of online pilot studies, because they report on a selected patient group who are open to online counselling and who might feel privileged to have access to this new service. $^{20,21}$ However, as stated by Hilgart et $a l^{20}$ when online counselling is used as an expansion to existing care services rather than as a replacement, the outcomes of patients who are open to adopting new technology are particularly relevant. We thus feel justified in being positive about their evaluations of telegenetics.

The major advantages of online counselling mentioned by our patients were time and cost savings, flexibility and being in their own, familiar environment. A substantial number of patients experienced technical problems, but only few reported these as a disadvantage of online counselling. The disadvantage of having less personal contact with the counsellor, mentioned afterwards by almost one-third of online patients, was surprisingly not reflected in their mean satisfaction scores afterwards. This could suggest that our patients do not see personal contact with their counsellor as an essential part of genetic counselling and thus find this not necessary to be satisfied.

The psychological outcomes (PPC and STAI) of our online patients did not differ significantly from controls regarding changes after counselling versus beforehand. Moreover, these outcomes are comparable to previous reports on PPC and STAI outcomes for in-person oncogenetic counselling. ${ }^{1,13,22-25}$ The mean anxiety level of online patients both before and after counselling was significantly lower than of controls. This might be an indication that patients who choose for online counselling are in general less anxious in nature than patients who choose for in-person counselling. Only three previous reports about online genetic counselling measured patient outcomes using validated standardized psychological measures, partly using the same as we did. ${ }^{1,5,6}$ Their usefulness as references for our outcomes is limited. Previous studies reported on the importance of an already established counselling relationship on performing an online counselling session. ${ }^{7,19,26}$ Although without such an existing relationship, our online patients reported to be satisfied and showed similar psychological outcomes as controls. Moreover, although these outcomes were not part of our evaluation study, no online patients did have an additional counselling session apart from their online pre-test session and the result sessions performed. Only one online patient, who turned out to be carrier of her familial mutation, had multiple contacts with one of the departments' social workers from about a year after testing. In the control group, one patient had a single contact with one of the departments' social workers and one patient reported having initiated contact with her occupational social worker, shortly after they both turned out to be carriers from the familial mutations they were tested for. In addition, we assessed the ways testing results were delivered for both patient groups, as part of checking the course of the counselling process. This did not reveal clear differences between both groups. Finally, the only previous study on online genetic counselling from patients' homes did not widely measure psychological patient outcomes. ${ }^{2}$ The home-based online counselling of our patients showed to be feasible: instructions and self-installation were well accepted and technical problems could at least partly be managed, mostly together with genetic professionals. Similar outcomes were reported previously by Meropol et al. ${ }^{2}$

Our study has some limitations: results can be biased by only including patients who were open to, and sufficiently equipped for, online counselling, and by comparison with a matched control group instead of doing a randomized controlled trial. Furthermore, the positive outcomes in our patient group cannot automatically be assumed on the longer term and for other presymptomatic and symptomatic patient groups; further, more longitudinal research, and in larger and other patient groups, is needed to draw more certain conclusions about the general quality and acceptability of online genetic counselling. Although, our online patients did not have additional counselling sessions compared with controls, cardio and oncogenetic patients do comprise a large part of referrals in many genetic clinics. Finally, the inclusion of prenatal couples in our study was problematic due to the different way and urgent nature of referrals.

To conclude, our predefined aims are met, suggesting online counselling as a valuable addition to existing in-person care for some of our patients. Patients who chose for online counselling were satisfied with it, even more than they expected beforehand, despite the current technical imperfections. Moreover, the psychological outcomes of online patients were comparable to controls. Evaluation of our telegenetics application from professionals' and economic perspectives will be logical next steps. Furthermore, technical improvements and cooperation with hospital ICT are needed to improve the quality of our online counselling for future patients and to allow for its implementation in regular patient care.

\section{CONFLICT OF INTEREST}

The authors declare no conflict of interest.

\section{ACKNOWLEDGEMENTS}

This pilot project was facilitated by grants from the Innovation Fund of the Dutch health insurers and the European Social Fund (2010ESFN630), and by cooperation of Jack van Gils and his company 'myCoachconnect'. We thank 
Theo van de Meer ('De Praktijk'), Margot Woolderink and all patients, counsellors and case managers who participated in this project. Jackie Senior edited the manuscript.

1 Zilliacus EM, Meiser B, Lobb EA, Kirk J, Warwick L, Tucker K: Are videoconferenced consultations as effective as face-to-face consultations for hereditary breast and ovarian cancer genetic counseling? Genet Med 2011; 13: 933-941.

2 Meropol NJ, Daly MB, Vig HS et al: Delivery of internet-based cancer genetic counseling services to patients' homes: a feasibility study. J Telemed Telecare 2011; 17: 36-40.

3 Abrams DJ, Geier MR: A comparison of patient satisfaction with telehealth and on-site consultations: a pilot study for prenatal genetic counseling. J Genet Couns 2006; 15: 199-205.

4 Stalker HJ, Wilson R, McCune H, Gonzalez J, Moffett M, Zori RT: Telegenetic medicine: improved access to services in an underserved area. J Telemed Telecare 2006; 12: 182-185.

5 Coelho JJ, Arnold A, Nayler J, Tischkowitz M, MacKay J: An assessment of the efficacy of cancer genetic counselling using real-time videoconferencing technology (telemedicine) compared to face-to-face consultations. Eur J Cancer 2005; 41: 2257-2261.

6 Gray J, Brain K, Iredale R, Alderman J, France E, Hughes H: Letter to the editor: a pilot study of telegenetics. J Telemed Telecare 2000; 6: 245-247.

7 Hawkins AK, Creighton S, Ho A, McManus B, Hayden MR: Providing predictive testing for Huntington disease via telehealth: results of a pilot study in British Columbia, Canada. Clin Genet 2013; 84: 60-64.

8 Hopper B, Buckman M, Edwards M: Evaluation of satisfaction of parents with the use of videoconferencing for a pediatric genetic consultation. Twin Res Hum Genet 2011; 14: 343-346.

9 d'Agincourt-Canning L, McGillivray B, Panabaker $\mathrm{K}$ et al: Evaluation of genetic counseling for hereditary cancer by videoconference in British Columbia. BC Med J 2008; 50: 554-559.

10 Lea DH, Johnson JL, Ellingwood S, Allan W, Patel A, Smith R: Telegenetics in Maine: successful clinical and educational service delivery model developed from a 3-year pilot project. Genet Med 2005; 7: 21-27.

11 Gattas MR, MacMillan JC, Meinecke I, Loane M, Wootton R: Telemedicine and clinical genetics: establishing a successful service. J Telemed Telecare 2001; 7 (suppl 2): 68-70.

12 Yip MP, Chang AM, Chan J, MacKenzie AE: Development of the telemedicine satisfaction questionnaire to evaluate patient satisfaction with telemedicine: a preliminary study. J Telemed Telecare 2003; 9: 46-50.
13 Smets EM, Pieterse AH, Aalfs CM, Ausems MG, van Dulmen AM: The perceived personal control (PPC) questionnaire as an outcome of genetic counseling: reliability and validity of the instrument. Am J Med Genet 2006; 140: 843-850.

14 Van der Bij AK, de Weerd S, Cikot RJ, Steegers EA, Braspenning JC: Validation of the Dutch short form of the state scale of the Spielberger State-Trait Anxiety Inventory: considerations for usage in screening outcomes. Community Genet 2003; 6: 84-87.

15 Marteau TM, Bekker H: The development of a six-item short-form of the state scale of the state scale of the Spielberger State-Trait Anxiety Inventory (STAI). Br J Clin Psychol 1992; 31: 301-306.

16 Zellerino B, Milligan SA, Brooks R, Freedenberg DL, Collingridge DS, Williams MS: Development, testing, and validation of a patient satisfaction questionnaire for use in the clinical genetics setting. Am J Med Genet C Semin Med Genet 2009; 151C: 191-199.

17 Gardner MR, Jenkins SM, O'Neil DA, Wood DL, Spurrier BR, Pruthi S: Perceptions of video-based appointments from the patient's home: a patient survey. Telemed J E Health 2015; 21: 281-285.

18 Van de Belt TH, Engelen LJ, Berben SA, Teerenstra S, Samson M, Schoonhoven L: Internet and social media for health-related information and communication in healthcare: preferences of the Dutch general populations. J of Med Internet Research 2013; 15: e220.

19 Zilliacus E, Meiser B, Lobb E, Duddung TE, Barlow-Stewart K, Tucker K: The virtual consultation: practitioners' experiences of genetic counseling by videoconferencing in Australia. Telemed J E Health 2010; 16: 350-357.

20 Hilgart JS, Hayward JA, Coles B, Iredale R: Telegenetics: a systematic review of telemedicine in genetics services. Genet Med 2012; 14: 765-776.

21 McDonald E, Lamb A, Grillo B, Lucas L, Miesfeldt S: Acceptability of telemedicine and other cancer genetic counseling models of service delivery in geographically remote settings. J Genet Counsel 2014; 23: 221-228.

22 Rothwell E, Kohlmann W, Jasperson K, Gammon A, Wong B, Kinney A: Patient outcomes associated with group and individual genetic counseling formats. Fam Cancer 2012; 11: 97-106.

23 Pieterse AH, van Dulmen AM, Beemer FA, Bensing JM, Ausems MG: Cancer genetic counseling: communication and counselees' post-visit satisfaction, cognitions, anxiety, and needs fulfillment. J Genet Couns 2007; 16: 85-96.

24 Brain K, Gray J, Norman P et al: Randomized trial of a specialist genetic assessment service for familial breast cancer. J Natl Cancer Inst 2000; 92: 1345-1351.

25 Bish A, Sutton S, Jacobs C, Levene S, Ramirez A, Hodgson S: Changes in psychological distress after cancer genetic counselling: a comparison of affected and unaffected women. Br J Cancer 2002; 86: 43-50.

26 Zilliacus E, Meiser B, Lobb E, Barlow-Stewart K, Tucker K: A balancing act - telehealth cancer genetics and practitioners' experiences of a triadic consultation. J Genet Counsel 2009; 18: 598-605.

Supplementary Information accompanies this paper on European Journal of Human Genetics website (http://www.nature.com/ejhg) 